\title{
A relação entre professores com sofrimento psíquico e crianças escolares com problemas de comportamento
}

\author{
The relation between teachers with psychological distress \\ and schoolchildren with behavior problems
}

\author{
GabrielaFranco Dias Lyra ${ }^{1}$ \\ Simone Gonçalves de Assis ${ }^{2}$ \\ Kathie N jaine ${ }^{2}$ \\ Raquel deVasconcellos Carvalhaes deO liveira ${ }^{3}$ \\ Thiago de Oliveira Pires ${ }^{2}$
}

\footnotetext{
${ }^{1}$ Instituto Fernandes

Figueira, Fundação Oswaldo

Cruz. Av. Rui Barbosa 716/

40 andar, Flamengo .

20550-020 Rio de Janeiro

RJ. gabriela@fiocruz.br

2 Centro Latino-

Americano deEstudos de

Violência eSaúdeJ orge

Careli, Escola Nacional de

SaúdePública, Fundação

Oswaldo Cruz.

3 Instituto de Pesquisa

Clínica Evandro Chagas,

Fundação Oswaldo Cruz.
}

Abstract The present article aims to analyze the relation between teachers suffering from psychological distress and school children with behavioral problems. A total of 372 children were evaluated by teachers through utilizing the Teachers Report Form (TRF). The teachers also answered a questionnaire in order to evaluate some personal characteristics and the presence of psychological distress (Self Reported Questionnaire, SRQ-20). The prevalence of psychological distress found was of $21.8 \%$. The results show that the teachers presenting psychological distress identified higher percentages of internalizing problems in the children.

Key words Psychological distress, Behavior problems, Children, TRF, SRQ-20
Resumo 0 presente estudo tem por objetivo analisar a relação entre professores com sofrimento psíquico e crianças escolares com problemas de comportamento. Um total de 151 professores avaliou a presença de problemas de comportamento em 372 alunos, através da escala Teacher's Report Form (TRF). Os alunos foram selecionados através de amostragem por conglomerados e estavam matriculados na primeira série do ensino fundamental. Os professores também responderam a um questionário auto-aplicado a fim de avaliar algumas características pessoais e a presença de sofrimento psíquico (Self Reported Q uestionnaireSRQ -20). A prevalência de sofrimento psíquico encontrada entre os professores foi de $21,8 \%$. Os resultados mostram percentuais mais elevados na identificação de problemas internalizantes pelas professoras que apre sentam sofrimento psíquico.

Palavras-chave Sofrimento psíquico, Problemas de comportamento em crianças, TRF, SRQ-20 


\section{Introdução}

0 presenteartigo tem como objetivo analisar a relação entre professores com sofrimento psíquico e a sua capacidade de identificar crianças com problemas de comportamento internalizantes, externalizantes ecom déficit deatenção e/ou hiperatividade.

A atuação docente, em especial de séries iniciais, traz importantes contribuições para o processo de desenvolvimento infantil. 0 longo tempo deconvivência estabelecido em sala deaula ea possibilidade de avaliar a criança na relação com outras da mesma faixa etária facilitam a observação do professor, conferindo-Ihe uma posição estraté gica na identificação de crianças que apresentam problemas de comportamento.

$\mathrm{Na}$ realidade brasileira, pouco se tem investigado sobre a criança e os possíveis problemas de comportamento que possam ocorrer nesta faixa etária. Em países mais desenvolvidos, estima-seque 10 a $20 \%$ das crianças apresentam algum problema de saúde mental, colocando-se entre as cinco principais causas de doença acima de cinco anos de idade ${ }^{1,2}$.

Estudo no Brasil mostrou resultados próximos aos de países desenvolvidos. I nvestigação com 1.251 crianças escolares de 7 a 14 anos, em Taubaté, São Paulo encontrou uma prevalência de crianças com problemas de comportamento de $12,5 \%$. Considerando os tipos de problemas, verificou-se que a maior freqüência ocorreu no grupo de crianças com transtornos de conduta, com estimativas em torno de 7\%. Em segundo, prevaleceram as crianças com transtornos de ansiedade, cuja ocorrência foi de $5,2 \%$. Os transtornos de déficit de atenção e/ou hiperatividade representaram 1,5\% das crianças estudadas 3 .

De uma maneira geral, os problemas de comportamento representam déficits ou excedentes comportamentais que prejudicam a interação da criança com os pares e adultos de sua convivência4. São freqüentemente divididos em problemas que se externalizam (sintomas de comportamento de quebrar regras e agressividade, tais como os transtornos de conduta) e problemas que se internalizam (tais como depressão e ansiedade) ${ }^{5}$.

Os comportamentos que se internalizam restringem-se ao âmbito privado da criança, não sendo diretamente dirigidos para o entorno em que esta vive. Já os comportamentos externalizantes interferem no cumprimento de tar efas evolutivas como as requeridas pela escola, por terem alta prevalência, prognóstico pobree por serem fatores de risco para inadaptação psicossocial na adolescência.

Além dessas categorias, destaca-se o transtor- no de déficit de atenção e/ou hiperatividade ${ }^{6}$, um padrão persistente de desatenção e/ou hiperatividade da criança, mais freqüente e severo do que aquele tipicamente observado em indivíduos em nível equivalente de desenvolvimento. Chae et al. ${ }^{7}$ relatam que a criança com déficit de atenção e/ou hiperatividade tem uma tendência a demonstrar comportamento agressivo, sofrer com baixa autoestima, envolver-seconstantementeem brigas, isolar-se em situações sociais e ter dificuldade de aprendizagem.

Crianças com problemas de comportamento apresentam modos de enfrentamento diferenciados frente às situações cotidianas e às relações interpessoais nas diversas esferas de convivência social. Em sala de aula, as principais manifestações nas crianças são os comportamentos tidos como socialmente inadequados ${ }^{4}$ e 0 baixo desempenho escolar ${ }^{8}$. Dependendo do tipo de problema, 0 aluno pode ser considerado desobediente, impaciente, agitado, destrutivo ou, então, desanimado, desacreditado e quieto, o que não, necessariamente, chama a atenção do professor.

Com base nessas considerações, pouco se tem debatido sobre a possibilidade de determinadas variáveis interferirem na visão que o professor tem sobre os problemas de comportamento dos alunos. Alguns estudos mostram que características dosalunos, tais como gênero, sexo econdição socioeconômica podem contribuir para o julgamento e tomadas de decisão dos professores sobre os comportamentos em sala de aula. N este sentido, há indícios que: crianças negras estão mais sujeitas a encaminhamentos para classes de educação especial do que crianças de outras raças'; meninos são avaliados como tendo mais problemas relacionados à leitura do que meninas ${ }^{10}$; crianças pertencentes a estratos socioeconômicos mais pobres são mais prováveis a serem indicadas para estratégias de intervenção em decorrência de problemas de fala ${ }^{11}$.

Características do professor também se constituem elementos importantes para a avaliação docente sobre os problemas de comportamentos dos alunos. 0 estresse vivenciado em sala de aula e a presença de problemas de saúde mental dos professores podem dificultar suas tomadas de decisão epossibilitar resultados nem semprepositivos para a criança ${ }^{12}$.

O sofrimento psíquico, também chamado transtorno psiquiátrico menor, caracteriza-se por um mal-estar inespecífico, com repercussões fisiológicas e psicológicas que podem acarretar limitações severas no dia-a-dia e pode se transformar em doença pela sua intensidade e cronicidade ${ }^{13}$. São problemas que não necessariamente originam a 
procura imediata por intervenção médica, mas que podem interferir nas relações e observações cotidianas do indivíduo.

Estatísticas sobre a presença de sofrimento psíquico dos docentes mostram percentuais sempre elevados, porém variados, segundo os diferentes contextos socioculturais. Pesquisas nacionais realizadas com o Self Reported Questionnaire (SRQ 20) encontraram taxas que variaram de $20 \%$ a $55,9 \%{ }^{14-17}$. Estudo com professores do ensino básico no Chileencontrou uma prevalência de $28,6 \%$, utilizando outra escala (General H ealth Questionnaire GHQ-20) ${ }^{18}$.

Diversos fatores podem estar relacionados à presença de sofrimento psíquico dos professores, tais como condições de vida e trabal ho desfavoráveis, desprestígio profissional, relações desiguais degênero edificuldades na vida familiar. Entretanto, a maioria dos estudos sobre sofrimento psíquico desta categoria tem debatido a influência do trabalho docente. Araújo et al. ${ }^{17}$, procurando investigar os fatores associados ao sofrimento psíquico dos professores da rede particular de Salvador, ressaltaram a importância das diversas características do processo de trabalho, tais como: atividade repetitiva, dificuldades de relações com os colegas de trabalho, insatisfação no desempenho das atividades, desgaste nas relações professor-aluno, ambiente intranqüilo e estressante, falta de autonomia no planejamento das atividades, ritmo acelerado de trabal ho, pressão da direção da escola e salas de aula inadequadas. Gasparini et al. ${ }^{19}$, avaliando professores do ensino fundamental da redemunicipal deBelo Horizonte, observaram uma forte associação entre transtornos psíquicos com experiência de violência na escola, percepção negativa sobre o trabalho e condições do ambientefísico laboral.

\section{M etodologia}

0 presenteartigo originou-sedeuma pesquisa longitudinal, iniciada em 2005, com quinhentas crianças e que teve por objetivo conhecer a magnitude dos problemas de comportamento dos alunos matriculados em escolas municipais da rede de ensino pública de São Gonçalo ${ }^{20}$. As crianças foram selecionadas por amostragem por conglomerado simples em três estágios de seleção: escolas (25); turmas (50) de $1^{a}$ série do $2^{\circ}$ segmento do $1^{10}$ ciclo ( antiga $1^{\mathrm{a}}$ série do ensino fundamental) ealunos ( 10 em cada uma das turmas). Os dados apresentados no artigo foram coletados na segunda fase da pesquisa (2006).
A análise apresentada neste artigo refere-se a 372 crianças aferidas por 151 professoras, em 83 escolas em que os alunos estudavam no ano de 2006. A redução do número de crianças avaliadas no presente artigo se deve a: a) não localização de 28 crianças; b) exclusão de dezesseis crianças em função do teste do quociente de inteligência (uma não realizou o teste equinze foram avaliadas como "intelectual mente deficientes", o queimpossibilitaa aferição de problemas de comportamento); c) preenchimento insuficientepelas professoras dosquestionários de quinze alunos; d) recusa de professoras em responder aos questionários de cinqüenta alunos; e) não retorno do instrumento de autoavaliação pelas professoras de dezenove crianças.

\section{Instrumentos}

0 Teacher's Report Form (TRF) ${ }^{21}$ éum instrumento desenvolvido para a avaliação de professores sobre crianças de 6 a 18 anos. É um questionário preenchido por professores, orientadores pedagógicos ou outros profissionais que participam do desenvolvimento escolar da criança e com condições de mostrar seu ponto de vista em relação ao comportamento apresentado pela criança na escola nos últimos dois meses. A versão utilizada da TRF é a traduzida para a cultura brasileira por Bordin et al. ${ }^{22}$ e ainda não há dados sobre confiabilidade e validade publicados. Todavia, a comparação dos problemas de comportamento aferidos pelos responsáveis $(C B C L)$ e professores (TRF) na primeira fase da pesquisa mostrou correlação com significância menor que $1 \%^{20}$, tal como observado em outros estudos internacionais $s^{23,24}$.

Exemplos de itens que aferem problemas de comportamento na TRF: preferência por ficar sozinho; recusar-se a falar; destruir objetos; ser infeliz, triste ou deprimido, desobediente, agitado, medroso ou ansioso demais. As opções de resposta variam de: falsa; pouco verdadeiro/às vezes verdadeiro; muito verdadei ro/freqüentementeverdadeiro. Possibilita aferir crianças sem problemas de comportamento (não clínicas) e com problemas em nível limítrofe ou clínicas (nos resultados deste artigo estas duas categorias estão agregadas).

Os questionários com a TRF foram colocados em envelope com o nome do aluno participante e entregues ao professor para a realização do preenchimento, devolvendo-os dias após. Além da TRF, um questionário auto-aplicado foi preenchido pelo professor a fim de verificar dados pessoais e a pre sença de problemas de saúde mental. Foram investigadas informações como: maior nível educa- 
cional do professor; tempo de experiência na docência; número de alunos em sua turma; número de escolas que trabalha; intervalo de tempo entre uma escola e outra, sem descanso algum; tempo semanal dos alunos sob seus cuidados.

A presença desofrimento psíquico dos professores foi aferida por meio do Self Reported Q uestionnaire - SRQ2025, que foi validado para o português por M ari eWilliams ${ }^{26}$. Exemplos de itens que compõem a SRQ-20: sentir-senervoso(a), tenso(a) ou agitado(a); sentir-se triste; sentir-se cansado(a) o tempo todo; ter dores de cabeça freqüentemente; ter dificuldade para realizar com satisfação suas atividades diárias, cansar-secom facilidade; ter falta de apetite; eter má digestão. É composta por itens que aferem sintomas somáticos de humor depressivo/ansioso, decréscimo de energia vital e pensamentos depressivos, segundo classificação sugerida por lacoponi e M ari27. O ponto de corte utilizado para o estabelecimento de suspeição de sofrimento psíquico foi de oito ou mais respostas positivas dentre as vinte perguntadas, seguindo o meIhor ponto de corte segundo $\mathrm{M}$ ari e Williams ${ }^{26}$.

Os dados que compõem o perfil das crianças, apresentados na Tabela 1, foram informados pelos responsáveis em outro instrumento que faz parte da pesquisa original. São eles: sexo, idade, distorção série idade de dois ou mais anos e cor da pele da criança, estrutura familiar e escolaridade materna. Do questionário materno, também foi obtida a Classificação Econômica Familiar, desenvolvida pela Associação Brasileira de Empresas de Pesquisa $(A B E P)^{28}$, para determinar as classes econômicas, segundo o poder de consumo da família. Dois grupos de classes sociais foram estabelecidos, segundo o escore total obtido: B eC (17 a 11 escores), $D$ e E ( 10 a 0 escores).

\section{Processamento eanálise dos dados}

0 instrumento de avaliação da criança pelo professor foi digitado einserido no programa Epidata versão 3.1. Para a análise de dados, foi utilizado o programa Statistical Package for Social Sciences SPSS versão 15. A associação entre presença de sofrimento psíquico dos professores e a identificação de problemas de comportamento nos alunos pelos professores foi analisada pelo teste de quiquadrado. A penas se apresentaram associações estatísticas significativas ao nível de $5 \%$.

A pesquisa foi aprovada pelo Comitê de Ética em Pesquisa da Escola Nacional de Saúde Pública/ Fiocruz eos professores ea direção das escolas assinaram termos de consentimento livree esclarecido.

\section{Resultados}

\section{Alunos}

Dentre as crianças estudadas, $51,1 \%$ eram meninas e $48,9 \%$, meninos. N oventa equatro por cento das crianças tinham até dez anos no momento da coleta de dados. Quanto à estrutura familiar, $52,5 \%$ residiam com o pai e mãe, ao passo que $24,6 \%$ viviam somente com um dos pais (a maioria com a mãe). Grande parte das mães tinha até 0 1 - grau $(71,8 \%)$ e $44 \%$ das famílias encontravamse no estrato social $\mathrm{D}$ e E. Em relação à distorção série/idade, 33,6\% das crianças apresentavam atraso escolar de dois ou mais anos (Tabela 1). Estes dados sinalizam as dificuldades socioeconômicas enfrentadas pelas famílias da rede pública municipal de ensino de São Gonçalo.

No que se refere aos problemas de comportamentos observados entre os alunos, tem-se na Tabela 2 que 0 transtorno mais apontado pelas professoras foi o problema externalizante(12,6\%), seguido pelos com a atenção e/ou hiperatividade $(10,8 \%)$ einternalizantes $(9,1 \%)$.

Tabela 1. Perfil das crianças e famílias da rede pública de ensino de São Gonçalo, RJ.

\begin{tabular}{|c|c|c|}
\hline & $\mathbf{N}$ & $\%$ \\
\hline \multicolumn{3}{|l|}{ Sexo } \\
\hline M asculino & 182 & 48,9 \\
\hline Feminino & 190 & 51,1 \\
\hline \multicolumn{3}{|l|}{ Cor } \\
\hline Branca & 127 & 34,8 \\
\hline Negra & 46 & 12,6 \\
\hline Parda & 192 & 52,6 \\
\hline \multicolumn{3}{|l|}{ Idade da criança } \\
\hline 7 a 8 anos & 168 & 48,0 \\
\hline 9 a 10 anos & 161 & 46,0 \\
\hline 11 anos ou mais & 21 & 6,0 \\
\hline \multicolumn{3}{|l|}{ Estrutura familiar } \\
\hline Pai e mãe & 188 & 52,5 \\
\hline Pai e madrasta/mãe e padrasto & 70 & 19,6 \\
\hline Só com um dos pais & 88 & 24,6 \\
\hline Sem pai e sem mãe & 12 & 3,4 \\
\hline \multicolumn{3}{|l|}{ Estrato social } \\
\hline$B+C$ & 173 & 56,0 \\
\hline $\mathrm{D}+\mathrm{E}$ & 136 & 44,0 \\
\hline \multicolumn{3}{|l|}{ Distorção série/idade } \\
\hline Não & 237 & 66,4 \\
\hline Sim & 120 & 33,6 \\
\hline \multicolumn{3}{|l|}{ Escolaridade materna } \\
\hline Não sabe ler e escrever & 21 & 6,0 \\
\hline $1^{\circ} \mathrm{grau}$ completo/ incompleto & 250 & 71,8 \\
\hline $2^{\circ}$ grau completo/ incompleto & 74 & 21,3 \\
\hline Superior completo/ incompleto & 3 & 0,9 \\
\hline
\end{tabular}




\section{Professores}

Todos os 151 professores entrevistados são do sexo feminino. Na Tabela 3, vê-se que a maioria das docentes encontra-se na faixa dos 30 aos 49 anos de idade $(63,4 \%)$. Quanto à escolaridade, a maioria (49,6\%) possui nível superior incompleto/completo, somado a 25,2\% com especialização. Boa parte das professoras pesquisadas demonstrou experiência na profissão. Todavia, 36\% têm menor tempo de atuação (nove anos ou menos).

Em relação ao número de escolas em que trabalhavam concomitantemente, $47,2 \%$ das professoras relataram trabalhar em duas ou mais escolas; $45,5 \%$ afirmaram que costumam sair de uma escola para trabalhar em outra imediatamente, sem descanso. Em relação ao tempo (horas semanais) que os alunos ficam em aula sob os seus cuidados, $45,5 \%$ ficam mais de vinte horas semanais. $M$ ais da metade das professoras $(53,6 \%)$ relatou ter mais de trinta alunos por turma. Esses dados apontam para a sobrecarga de trabal ho e para a necessidade de continuada atenção a muitas crianças por longos períodos de tempo.

Em relação à saúde mental das professoras, $21,8 \%$ relataram sofrimento psíquico. No Gráfico 1 , vêem-se os itens respondidos pelas professoras agregados segundo a classe de sintomas apresentados. Nota-se que alguns sintomas destacam-se dos demais: sentir-se nervosa, tensa ou agitada $(54,7 \%)$, encontrar dificuldades para realizar com satisfação suas atividades diárias (41,3\%), dormir mal $(43,5 \%)$ eter dores de cabeça freqüentemente $(38,4 \%)$. Vale a pena ressaltar a importância dos pensamentos depressivos, embora em menores

Tabela 2. Prevalência de problemas de comportamento em crianças de São Gonçalo, RJ .

\begin{tabular}{lrrrr}
\hline & $\mathrm{N}$ & $\%$ & \multicolumn{2}{c}{ I.C. $95 \%$} \\
\hline $\begin{array}{l}\text { Problemas com atenção/ } \\
\text { hiperatividade }\end{array}$ & & & & \\
$\quad$ N ão & 332 & 89,2 & 85,6 & 92,2 \\
$\quad$ Sim & 40 & 10,8 & 7,8 & 14,4 \\
Internalizante & & & & \\
$\quad$ N ão & 338 & 90,9 & 87,5 & 93,6 \\
$\quad$ Sim & 34 & 9,1 & 6,4 & 12,5 \\
Externalizante & & & & \\
$\quad$ Não & 325 & 87,4 & 83,6 & 90,6 \\
$\quad$ Sim & 47 & 12,6 & 9,4 & 16,4 \\
\hline & & & &
\end{tabular}

percentuais: $5,8 \%$ das professoras se sentem uma pessoa inútil, sem préstimo, 3,6\% têm tido a idéia de acabar com a vida e 2,2\% se sentem incapazes de desempenhar um papel útil em sua vida.

\section{Saúde mental das professoras e identificação} de problemas de comportamento nos alunos

$\mathrm{Na}$ Tabela 4, observa-se que professoras que vivenciam sofrimento psíquico, comparadas às que não apresentam o transtorno, identificam mais problemas internalizantes em seus alunos ( $15,2 \%$ versus 7,3\%, respectivamente; $p$-valor $=0,042$ ).

Em relação aos problemas com a aten ção e/ou hiperatividade, encontra-se tendência similar, porém o nível designificância fica apenas próximo ao patamar estipulado no estudo. Para os problemas externalizantes, os resultados mostram-se menos incisivos.

Tabela 3. Perfil das professoras estudadas no ano de 2006 na rede pública de São Gonçalo, RJ.

\begin{tabular}{|c|c|c|}
\hline & $\mathrm{N}$ & $\%$ \\
\hline \multicolumn{3}{|l|}{ Faixa etária } \\
\hline 19-29 anos & 28 & 20,9 \\
\hline 30-49 anos & 85 & 63,4 \\
\hline 50 e mais & 21 & 15,7 \\
\hline \multicolumn{3}{|l|}{ M aior grau de formação escolar } \\
\hline 2o grau & 35 & 25,2 \\
\hline Superior incompletolcompleto & 69 & 49,6 \\
\hline Especialização & 35 & 25,2 \\
\hline \multicolumn{3}{|c|}{ Tempo (anos) que trabalha como professora } \\
\hline De 0 a 9 anos & 50 & 36,0 \\
\hline De 10 a 19 anos & 38 & 27,3 \\
\hline 20 anos ou mais & 51 & 36,7 \\
\hline \multicolumn{3}{|c|}{ Número de escolas por semana em que dá aula } \\
\hline Uma & 66 & 52,8 \\
\hline 2 ou mais & 59 & 47,2 \\
\hline \multicolumn{3}{|c|}{$\begin{array}{l}\text { H ábito de sair de uma escola para trabalhar em } \\
\text { outra escola imediatamente, sem descanso }\end{array}$} \\
\hline Sim & 56 & 45,5 \\
\hline Não & 67 & 54,5 \\
\hline \multicolumn{3}{|c|}{$\begin{array}{l}\text { Tempo (horas) por semana que os alunos ficam } \\
\text { em aula sob os cuidados do professor }\end{array}$} \\
\hline Até $20 \mathrm{~h}$ & 72 & 54,5 \\
\hline Mais de $20 \mathrm{~h}$ & 60 & 45,5 \\
\hline \multicolumn{3}{|l|}{ N úmero de alunos médio por turma } \\
\hline Até 30 alunos & 64 & 46,4 \\
\hline 31 alunos ou mais & 74 & 53,6 \\
\hline
\end{tabular}




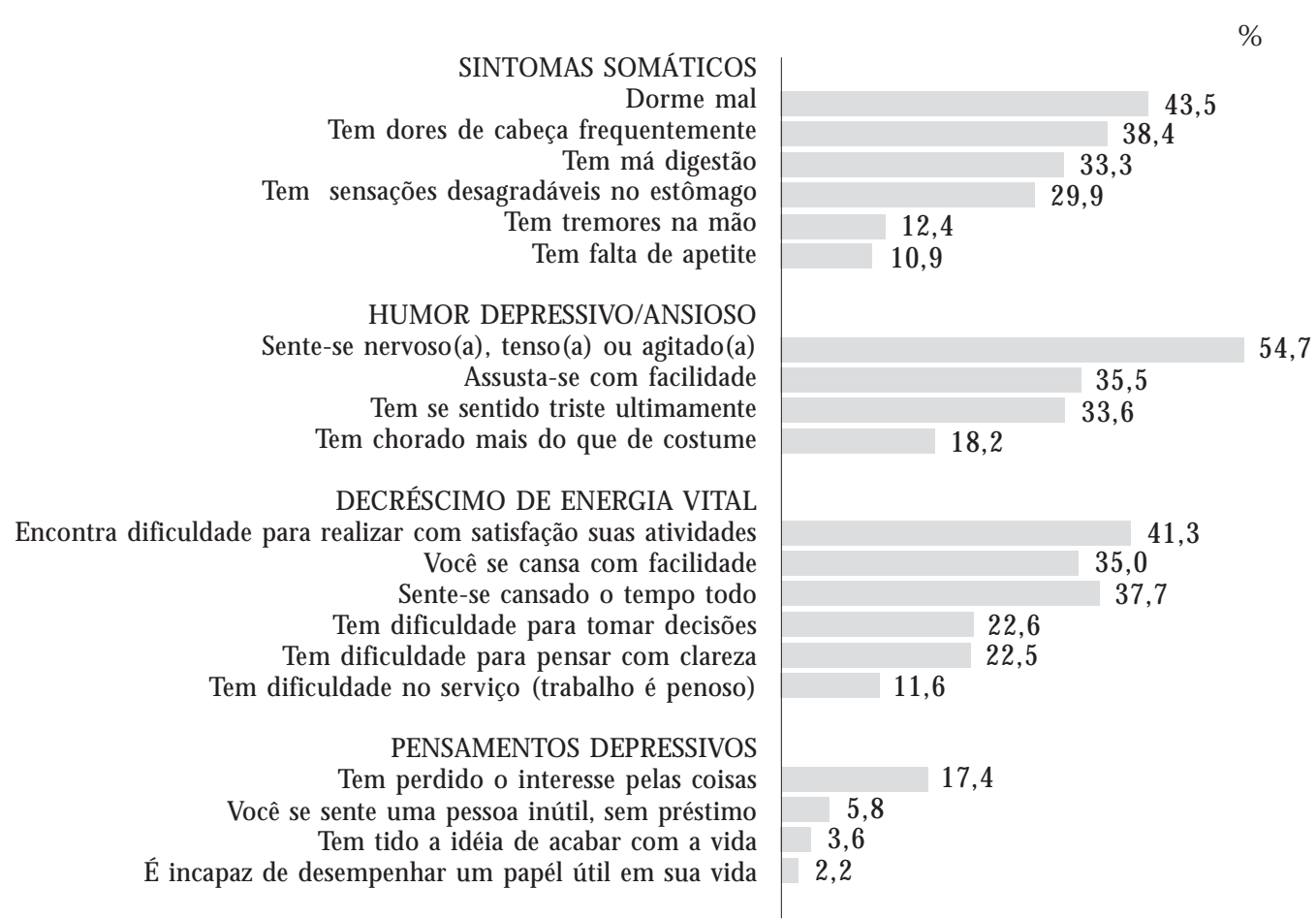

Gráfico 1. Sofrimento psíquico de educadoras de São Gonçalo, RJ (itens da SRQ20) (N=151).

Tabela 4. Associação entre sofrimento psíquico das professoras e identificação de problemas de comportamento de crianças.

\begin{tabular}{|c|c|c|c|c|}
\hline & & Não & Sim & P-valor \\
\hline \multicolumn{2}{|c|}{$\begin{array}{l}\text { Problemas com atenção/ } \\
\text { hiperatividade }\end{array}$} & & & 0,072 \\
\hline \multirow[t]{2}{*}{ Não } & $\mathrm{N}$ & 250 & 55 & \\
\hline & $\%$ & 90,9 & 83,3 & \\
\hline \multirow[t]{2}{*}{ Sim } & $\mathrm{N}$ & 25 & 11 & \\
\hline & $\%$ & 9,1 & 16,7 & \\
\hline \multicolumn{2}{|c|}{ Internalizante } & & & 0,042 \\
\hline \multirow[t]{2}{*}{ Não } & $\mathrm{N}$ & 255 & 56 & \\
\hline & $\%$ & 92,7 & 84,8 & \\
\hline \multirow[t]{2}{*}{ Sim } & $\mathrm{N}$ & 20 & 10 & \\
\hline & $\%$ & 7,3 & 15,2 & \\
\hline \multicolumn{2}{|c|}{ Externalizante } & & & 0,129 \\
\hline \multirow[t]{2}{*}{ Não } & $\mathrm{N}$ & 244 & 54 & \\
\hline & $\%$ & 88,7 & 81,8 & \\
\hline \multirow[t]{2}{*}{ Sim } & $\mathrm{N}$ & 31 & 12 & \\
\hline & $\%$ & 11,3 & 18,2 & \\
\hline
\end{tabular}

\section{Discussão}

A prevalência de $21,8 \%$ de professoras com sofrimento psíquico encontrada no presente artigo se aproxima da relatada em pesquisa realizada por Araújo et al. ${ }^{17}$, que utilizaram a mesma escala no cenário nacional, cuja taxa foi de 20,1\%. Comparada a outras categorias profissionais, observa-se realidade um pouco distinta. Na categoria profissional de bancários do Rio de Janeiro, verificam-se prevalências de sofrimento psíquico de $11,5 \%$ entre homens e $19,8 \%$ entre mulheres ${ }^{29}$. Utilizando como contraponto uma categoria profissional muito exposta à tensão no trabalho, tem-se que $35,7 \%$ dos policiais militares da cidade do Rio de Janeiro e $25,8 \%$ dos policiais civis da mesma cidade apresentam sofrimento psíquico medido pela SRQ $-20^{30}$.

Alguns autores apontam uma elevada taxa de sofrimento psíquico dos professores, acima das encontradas neste artigo, tais como a pesquisa de Reis et al. ${ }^{15}$, que encontraram uma prevalência de $55,9 \%$ dos professores da rede municipal de Vitó- 
ria da Conquista, ea deD el cor et al. ${ }^{16}$, que identificaram a presença do transtorno em $41,5 \%$ dos professores da rede particular de Vitória da Conquista. Ambos os estudos, realizados na rede pública e particular, utilizaram o SRQ -20. Um estudo conduzido por Gasparini et al. ${ }^{19}$ (utilizando a $\mathrm{Ge}$ neral Health Questionnaire GH Q-20) encontrou uma prevalência de $50,3 \%$ dos professores da rede municipal deBelo Horizonte.

Em relação ao grupo de sintomas aferidos, embora a SRQ-20 se limite a detectar os níveis de alteração do funcionamento psíquico enão a realizar diagnóstico, é possível observar algumas tendências também avaliadas em outros estudos ${ }^{15,17}$, como, por exemplo, uma maior manifestação de professoras com sintomas relacionados ao humor depressivo (se sentir nervoso, tenso ou agitado; assustar-se com facilidade; se sentir triste ultimamente) e aos sintomas somáticos (dormir mal; ter dores de cabeça freqüentemente; ter sensações desagradáveis no estômago).

Alguns fatores dificultam a comparação dos resultados entre as prevalências encontradas nos diferentes contextos. D estacam-se: diferentes escalas de aferição de transtornos mentais; variados pontos de corte utilizados para definição de distúrbio psíquico na SRQ-20; diversidade das populações e cenários estudados. Porto et al. ${ }^{14}$, ao detectar uma prevalência de sofrimento psíquico de $44 \%$ dos professores de escolas públicas e particulares de Vitória da Conquista, explicam que o período de realização da pesquisa é mais um fator que poderia contribuir para o aumento dos resultados encontrados, uma vez que os sintomas de sofrimento psíquico são transitórios e existem períodos escolares em que a carga da atividade docente aumenta e, conseqüentemente, potencializa os fatores estressantes para o professor.

Outro dado a ser levado em conta é a localização regional. Uma pesquisa nacional com 30.000 professores mostrou que características econômicas ou socioculturais se relacionam à variação nas taxas de problemas rel acionados à saúde mental do professor. Esteestudo revelou que as taxas de exaustão emocional dos professores variaram de $17 \%$ em M inas Gerais e 39\% no Rio Grande do Sul ${ }^{31}$.

A relação de transtornos emocionais com gê-

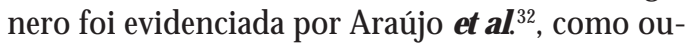
tro fator a ser levado em conta: professoras teriam prevalências mais altas do que professores. As inúmeras jornadas de trabalho que as mulheres estão submetidas e a sobrecarga doméstica, proporcionada pelas tarefas no cuidado com os filhos e com a casa, as tornariam mais vulneráveis à presença de sofrimento psíquico.
As características pessoais e do trabalho das professoras investigadas foram semelhantes às de estudos que encontraram relação entre sofrimento psíquico e características do trabalho docente ${ }^{15,16}$. O número grande de alunos por turma, evidenciado em $53,6 \%$ dos professores, bem como 0 número considerável de escolas que as professoras trabalham, relatado por $47,2 \%$ das professoras, são fatores que contribuem para o desgaste mental desta população. 0 docente, muitas vezes, dá aula em inúmeras escolas durante a semana, sem ao menos ter tempo para descansar durante essas atividades, como visto em $45,5 \%$ na população estudada. Também cabe ressaltar as outras jornadas de trabalho que muitas professoras realizam após a atividade docente, tendo em vista 0 papel social determinado à mulher de cuidadora da família.

A partir dos resultados observados entre os alunos, vale apontar que os problemas de comportamentos constituem-se um desafio para os professores em sala de aula. Como mostrado na pesquisa que deu origem a este estudo, cerca de $10 \%$ dos alunos apresentavam sinais de algum problema de comportamento, em nível clínico, segundo os professores, e este resultado esteve próximo ao detectado pelos pais na mesma pesquisa (cerca de $11 \%)^{20}$.

Massola e Silvares ${ }^{33}$ revelaram que os professores são capazes de identificar de forma adequada o potencial e os problemas de seus alunos, tal como fizeram pais e psicólogos treinados. Youngstrom et al..$^{34}$, em seu estudo com jovens americanos, lembram que os relatos dos professores são mais fidedignos em relação aos auto-relatos feitos por jovens para avaliação de comportamentos de hiperatividade, desatenção e comportamento opositor. Porém, ressaltam que, quanto aos comportamentos internalizantes, a auto-avaliação dos jovens é melhor do que a efetuada pelos professores.

Quanto aos tipos de problemas, os professores, de uma maneira geral, identificam mais alunos com problemas externalizantes do que com a atenção e/ou hiperatividadeeinternalizantes, fato também evidenciado em outros estudos ${ }^{34,35}$. I sso é mais provável de acontecer porque problemas externalizantes são mais visíveis aos olhos do professor e difíceis deadministrar em situações de sala deaula. Em relação aos problemas internalizantes, parece existir uma tendência de subestimar a severidade do impacto desses problemas. Na mesma direção, grande parte dos alunos com sintomas depressivos tende a se aproximar da figura de um aluno "normal" e que chama menos atenção em sala de aula $a^{36}$. 
Verificou-se que a presença de sofrimento psíquico associa-se à diferente visão dos professores em relação aos problemas internalizantes e bem próximo da significância estatística no que se refere aos problemas externalizantes. Estariam as professoras que apresentaram sofrimento psíquico sendo mais sensíveis aos sinais manifestados pelos alunos em sala de aula? Embora não se possa afirmar ou negar esta indagação a partir dos resultados deste artigo, é possível afirmar a existência de uma relação entre transtorno emocional das professoras e a identificação de problemas na saúde mental de crianças.

Alguns estudos sobre depressão materna ajudam a entender a complexidade existente entre a saúde mental do adulto e sua influência na avaliação de problemas emocionais e/ou comportamentais na criança ou no adolescente $\mathrm{e}^{37-39}$. Berg-Nielsen et al. ${ }^{40}$, utilizando instrumentos análogos ao TRF (Child Behaviour Checklist- CBCL e Youth Self Report - YSR), mostraram que mães com depressão tenderam a relatar mais problemas internalizantes comparadas aos adolescentes. Boylee Pickles ${ }^{37}$ confirmam que mães com sintomas de depressão informam mais problemas de comportamento de seus filhos em relação à avaliação dos professores.

Para Najmam et al. ${ }^{38}$, existem interpretações distintas para este fenômeno: mães com dificuldades emocionais tendem a superestimar os problemas de comportamento de seus filhos; mães que não são comprometidas emocionalmente podem subestimar os problemas de comportamentos das crianças. Para o autor, é difícil determinar qual dessas interpretações é a mais provável. Uma possibilidade é que pessoas deprimidas seriam mais sensíveis e precisas em suas percepções do que pessoas não-deprimidas ${ }^{41}$.

\section{Colaboradores}

GFD Lyra, SG de Assis, K Njaine, RVC de Oliveira e TO Pires participaram igualmente de todas as etapas da elaboração do artigo.
Como limitação do estudo, temos o fato de ser um estudo seccional, que não permite conhecer 0 nexo causal entretranstornos emocionais dos professores e os problemas de comportamento dos alunos. Também está restrito a uma população mais pobre, não permitindo avaliar diferenças entre estratos sociais mais díspares. Cabe ainda ressaltar que a amostra não é representativa dos professores da rede pública de São Gonçalo (e sim das crianças).

Finalizando, ressalta-se que a identificação de problemas de comportamento é condição básica para o bem-estar futuro da criança, mas por si só não traz efeitos benéficos para uma intervenção adequada. A escassez de recursos materiais e humanos e as dificuldades relacionais observadas em muitas escolas contribuem para que os professores se sintam impotentes perante situações com as quais não sabem lidar, a exemplo dos alunos com problemas emocionais. A falta de rede de apoio social e de atendimento em saúde mental reduz ainda mais a expectativa dos docentes no cuidado das crianças com dificuldades de comportamento.

A capacitação dos professores na identificação e no encaminhamento de crianças em idade escolar com problemas de comportamento poderá contribuir para uma intervenção precocee, conseqüentemente, um encaminhamento a uma rede de apoio que dê o suporte necessário para a resolução dos problemas, uma vez que tais situações podem prejudicar o desenvolvimento infantil e a vida adulta da criança. Por outro lado, o apoio emocional aos professores com sofrimento psíquico e a todos que trabalham em condições tão desfavoráveis éigualmentenecessário para seconstruir uma escola mais digna ao crescimento e desenvolvimento infantil. 
1. Bird HR. Epidemiology of Childhood Disorders in a Cross-Cultural Context. J Child Psychol Psychiatry 1996; 37(1):35-49.

2. Murray CJL, Lopez AD, editors. The Global Burden of Disease. Geneva: World Health Organization; 1996.

3. Fleitlich-Bilyk B, Goodman R. Prevalence of child and adolescent psychiatric disorders in Southeast Brazil. J American Academy of Child \& Adolescent Psychiatry 2004; 43(6):727-734.

4. Silva ATB. Problemas de comportamento e comportamentos socialmente adequados: sua relação com as habilidades sociais educativas de pais [dissertação]. São Carlos (SP): Universidade Federal de São Carlos; 2000.

5. Bee H. A criança em desenvolvimento. Porto Alegre: Artmed; 1996.

6. American Psychiatric Association. DSM IV: manual diagnóstico e estatístico de transtornos mentais. Porto Alegre: Artes M édicas; 1995.

7. Chae PK, Jung HO, Noh KS. Attention deficit hyperactivity disorder in Korean juvenile delinquents. Adolescence 2001; 36(144):707-725.

8. Santos PL, Graminha SSV. Problemas emocionais e comportamentais associados ao baixo rendimento acadêmico. Estudos de Psicologia 2006; 11(1):101-109.

9. Lanier J, Wittmer J. Teacher prejudice in referral of students to EM R programs. The School Counselor 1977; 24(3):165-170.

10. Rutter M, Caspi A, Fergusson D, Horwood IJ, Goodman $R, M$ aughan $B, M$ offitt TE, M eltzer $H$, Carroll J. Sex differences in developmental reading disability: new findings from 4 epidemiological studies. Journal of American M edical Association 2004; 29(16):2007-2012.

11. Franklin K, Grossman FM. Bias Effects of Socioeconomic Status and Sex in Decision Making in SpeechLanguage Pathology. Journal of Speech and Hearing Disorders 1990; 55:82-89.

12. Egyed CJ, Short RJ. Teacher self-efficacy, burnout, experience and decision to refer a disruptive student. School Psychology International 2006; 27:462-474.

13. Coutinho ESF. Fatores sócio-demográficos e morbidade psiquiátrica menor: homogeneidade e heterogeneidade de efeitos [tese]. Salvador (BA): Universidade Federal da Bahia; 1995.

14. Porto LA, Carvalho FM, Oliveira NF, Neto M AS, Araújo TM, Reis EJFB, Delcor NS. Associação entre distúrbios psíquicos e aspectos psicossociais do trabalho de professores. Rev. Saúde Pública 2006; 40(5):818-826.

15. Reis EJFB, Carvalho FM, Araújo TM, Porto LA, N eto MAS. Trabalho e distúrbios psíquicos em professores da rede municipal de Vitória da Conquista, Bahia Brasil. Cad Saúde Pública 2005; 21(5):1480-1490.

16. Delcor NS, Araújo, TM, Reis EJFB, Porto LA, CarvaIho FM, Silva MO, Barbalho L, Andrade MA. Condições de trabalho e saúde dos professores da rede particular de ensino de Vitória da Conquista, Bahia, Brasil. Cad Saúde Pública 2004; 20(1):187-196.

17. Araujo TM, Neto AMS, Reis EJFB, Dutra FRD, Azi $\mathrm{GR}$, Alves RL. Trabalho docente e sofrimento psíquico: um estudo entre professores de escolas particulares de Salvador, Bahia. Rev. FAEEBA - educ. contemp. 2003; 12(20):485-495.
18. Claro TS, Bedregal GP. Aproximacion al estado de salud mental del professorado en 12 escuelas de Puente Alto, Santiago, Chile. Rev. M éd. Chile. 2003; 131(2):159-167.

19. Gasparini SM, Barreto SM, Assunção AA. Prevalência de transtornos mentais comuns em professores da rede municipal de Belo Horizonte, M inas Gerais, Brasil. Cad. Saúde Pública 2006; 22(12):2679-2691.

20. Assis SG. A violência familiar produzindo reversos. Problemas de comportamento em crianças escolares de São Gonçalo - Rio de Janeiro [relatório final de pesquisa]. Rio de Janeiro: Fiocruz; 2007.

21. Achenbach TM. M anual for the Teacher's Report Form and 1991 Profile. Burlington, VT: University of Vermont/D epartment of Psychiatry; 1991.

22. Bordin IAS, M ari JJ, Caeiro M F. Validação da versão brasileira do Child Behavior Checklist (CBCL) (Inventário de comportamentos da Infância e Adolescência): dados preliminares. Revista ABP-APAL 1995;17(2):55-66.

23. Ferdinand RF, Van Der Ende J, Verhulst FC. ParentTeacher Disagreement Regarding Psychopathology in Children: A Risk Factor for Adverse Outcome? Acta Psychiatr Scand 2007; 115:48-55.

24. Kolko DJ, Kazdin AE. Emotional/behavioral problems in clinic and nonclinic children: correspondence among child, parent and teacher reports. J Child Psychol Psychiatry 1993; 34(6): 991-1006.

25. Harding TW. M ental Disorder in Primary Health Care: A Study of Their Frequency and Diagnosis in Four Developing Countries. Psychological M edicine 1980; 10:231-241.

26. Mari JJ, Williams P. A validity study of a psychiatric screening questionnaire (SRQ20) in primary care in the city of São Paulo. British Journal of Psychiatry 1986; 148:23-26

27. Iacoponi E, Mari JJ. Reliability and factor structure of the Portuguese version of Self-Reporting Questionnaire. International Journal of Social Psychiatry 1998; 35(3):213-222.

28. Associação Brasileira de Empresas de Pesquisa. Critério de classificação econômica Brasil [site na Internet]; 2003. [acessado 2008 set 20]. Disponível em: http://www.abep.org/codigosguias/ ABEP CCEB.pdf

29. Beltrão IK, Duchiade M P, Chor D, Valente J, Fonseca MMJ, Andrade CR, Lima JM. Pesquisa de saúde dos associados da CASSI [relatório final]. Rio de Janeiro: Ence-FIBGE/Ensp-Fiocruz; 1996.

30. Minayo MCS, Souza ER, Constantino P. Missão prevenir e proteger. condições de vida, trabalho e saúde dos Policiais Militares do Rio de Janeiro. Rio de Janeiro: Fiocruz; 2008.

31. Codo W, coordenador. Educação: carinho e trabalho. 4a ed. Petrópolis: Vozes; 2006.

32. Araujo TM, Godinho TM, Reis EJFB, Almeida M MG. Diferenciais de Gênero no trabalho docente e repercussões sobre a saúde. Cien Saude Colet 2006; 11(4):1117-1129.

33. M assola GM , Silvares EFM. A percepção do distúrbio de comportamento infantil por agentes sociais versus encaminhamento para atendimento psicoterapêutico. Revista Interamericana de Psicologia 2005; 39(1):139-150. 
34. Youngstrom E, Loeber R, Stouthamer-Loeber M. Patterns and correlates of agree between parent, teacher and male adolescent ratings of externalizing and internalizing problems. Journal of Consulting $\&$ Clinical Psychology 2000; 68(6):1038-1050.

35. Verhulst FC, Dekker MC, Van Der Ende J. Parent, teacher and self-reports as predictors of signs of disturbance in adolescents: whose information carries the most weight?Acta Psychiatr Scand 1997; 96(1):75-81.

36. Avanci JQ. Depressão em crianças. Série Violência e Saúde M ental infanto-juvenil. Rio de Janeiro: Fiocruz/EN SP/CLAVES/CN PQ; 2008.

37. Boyle $M H$, Pickles A. Maternal depressive symptoms and ratings of emotional disorder symptoms in children and adolescents. J Child Psychol Psychiat 1997; 38(8):981-992.

38. Najman JM, Williams GM, Nikles J, Spence $S$, Bor W, O'Callaghan M, Le Brocque R, Andersen MJ, Shuttlewood GJ. Bias influencing maternal reports of child behaviour and emotional state. Soc Psychiatry Psychiatr Epidemiol 2001; 36:186-194.

39. Fergusson DM, Lynskey MT, Horwood LJ. The effects of maternal depression on maternal ratings of child behaviour. Journal of Abnormal Child Psychology 1993; 2:245-269.

40. Berg-Nielsen TS, Vika A, Dahl AA. When adolescents disagree with their mothers: CBCL-YSR discrepancies related to maternal depression and adolescent self-esteem. Child: Care, Health \& Development 2003; 29(3):207-213.

41. Ackermann R, DeRubeis RJ. Is depressive realism real? Clin Psychol Review 1991; 11:565-584.

Artigo apresentado em 28/04/2008

Aprovado em 22/08/2008

Versão final apresentada em 07/10/2008 